\title{
ASYMPTOTIC ESTIMATES OF VISCOELASTIC GREEN'S FUNCTIONS NEAR THE WAVEFRONT
}

\author{
BY
}

\author{
ANDRZEJ HANYGA
}

ul. Bitwy Warszawskiej 1920r. 14/52, Warszawa, Poland

\begin{abstract}
Asymptotic behavior of viscoelastic Green's functions near the wavefront is expressed in terms of a causal function $g(t)$ defined in Hanyga and Seredyńska (2012) in connection with the Kramers-Kronig dispersion relations. Viscoelastic Green's functions exhibit a discontinuity at the wavefront if $g(0)<\infty$. Estimates of continuous and discontinuous viscoelastic Green's functions near the wavefront are obtained.
\end{abstract}

\section{Notation.}

$\begin{array}{lll}\mathrm{D}^{n} f(t) & \mathrm{d}^{n} f(t) / \mathrm{d} t^{n} & \\ f * g & \text { Volterra convolution } & \int_{0}^{t} f(s) g(t-s) \mathrm{d} s \\ \mathcal{L}(f)=\tilde{f} & \text { Laplace transform of } \quad & \int_{0}^{\infty} \mathrm{e}^{-p t} f(t) \mathrm{d} t \\ ] a, b] & \text { the set } a<x \leq b & \\ \mathfrak{R} z, \mathfrak{I} z & \text { real and imaginary part of } z & \\ J(t), J^{\prime}(t) & \text { creep compliance, creep rate } & \end{array}$

1. Introduction. It is fair to say that seismology and particularly seismic inversion theory is implicitly based on the assumptions that the medium supports discontinuity waves. This assumption ensures that seismic signals travel with the wavefront speed while retaining their shape except for scaling. However many published explicit models of viscoelastic media such as the fractional Zener models [2, 22, 24, 31] used in, among others, seismology, as well as the Cole-Davidson and Havriliak-Negami models [10, 17, used for modelling mechanical response of polymers, all lack this property. All these models are characterized by asymptotic power law behavior of the attenuation function (i.e. the logarithmic attenuation per unit propagation distance expressed as a function of circular frequency) in the high-frequency range, which entails continuity of the viscoelastic Green's function and all its temporal and spatial derivatives at the wavefront. A viscoelastic pulse in such a medium is preceded by a pedestal, i.e. a flat precursor

Received January 29, 2014.

2010 Mathematics Subject Classification. Primary 74D05.

Key words and phrases. Viscoelasticity, wavefront, attenuation, dispersion, shock wave, Bernstein function.

E-mail address: ajhbergen@yahoo.com 
that follows immediately after the wavefront [14,29] and therefore travels at a slower effective speed depending on the propagation time. (We ignore here the seismological and acoustic models in which signals propagate at infinite speeds [3,20,28.). A seismic signal travels with the wavefront if the wavefront of Green's function supports a discontinuity of the stress-velocity field and lags behind the wavefront if Green's function exhibits the pedestal effect [14. Relevance of the pedestal effect for seismic inversion was demonstrated in 13 and for borehole velocity surveys in [30.

Seismological literature, however, knows two exceptional models which are consistent with the assumptions made in seismology: the Jeffreys-Lomnitz creep and a creep model due to Strick and Mainardi. In the Jeffreys-Lomnitz and Strick-Mainardi viscoelastic media attenuation is bounded in the high-frequency range, and discontinuities are not immediately smoothed out. The last-mentioned models are considered in some detail in [12. The results obtained in this paper apply to both kinds of viscoelastic media. Besides we establish here a new criterion for existence of discontinuity waves.

In [16] and [10] a theory of attenuation and dispersion in general viscoelastic media with completely monotone relaxation moduli (or, equivalently, with creep compliances that are Bernstein functions) was developed. An application of this theory to the study of the relation between the high-frequency asymptotics of the attenuation function and the wavefront singularities of Green's function of an acoustic equation was made in [11. These results carry over to the viscoelastic equations of motion in one-dimensional space without any modification. It was shown in [1] that Green's functions with an unbounded attenuation function do not exhibit wavefront discontinuities, while those with an attenuation function increasing faster than the logarithmic rate in the high-frequency range exhibit the pedestal effect. If the attenuation function grows at the logarithmic rate in the high-frequency range, then the regularity of the wavefront gradually increases with time.

Related results have been obtained in earlier papers. Lokshin 21. proved that viscoelastic Green's functions are infinitely differentiable at the wavefront if $J^{\prime}(t) /|\ln (t)|$ is bounded or tends to infinity as $t \rightarrow 0$. Desch and Grimmer obtained the same result if the relaxation kernel has a logarithmic or stronger singularity [6]. The same authors showed that in the gap between boundedness and logarithmic singularity more complex regularity patterns are observed, such as stepwise regularization [7,11,18, which we shall also demonstrate here.

In this paper we shall use a causal function underlying the Kramers-Kronig dispersion relations [10,16] to study the behavior of viscoelastic Green's functions near the wavefront in more detail. In particular we shall derive a well-known criterion for existence of shock waves in viscoelastic media. This criterion probably also applies to nonlinear viscoelastic media under some assumptions on the nature of non-linearity [8,26]. The new approach gives more detailed information about the wavefront asymptotics of Green's functions. For example, for discontinuous Green's functions it provides information about the magnitude of the wavefront jump discontinuity as well as about the behavior of Green's function immediately behind the wavefront.

In [10,16] a causal function $g$ of physical dimension $1 / \mathrm{L}$ was introduced in such a way that the attenuation function $\mathcal{A}(\omega)$ is equal to the real part of $(-\mathrm{i} \omega) \tilde{g}(-\mathrm{i} \omega)$, while the 
dispersion function $\mathcal{D}(\omega):=\omega\left(1 / c(\omega)-1 / c_{0}\right)$ is given by the expression $\mathfrak{I}[\mathrm{i} \omega \tilde{g}(-\mathrm{i} \omega)]$. $c(\omega)$ denotes here the phase speed of a wavefield oscillating with the circular frequency $\omega$. Existence of the function $g$ is thus linked to the validity of the Kramers-Kronig dispersion relations.

We shall show here that the function $-g\left(t-|x| / c_{0}\right)|x|$ is an asymptotic phase function near the wavefront of viscoelastic Green's functions, where $c_{0}:=\sup _{\omega} c(\omega)$ is the wavefront speed and it is assumed that $c_{0}<\infty$. The theory is based on the assumption that the relaxation modulus is locally integrable and completely monotonic (LICM). This assumption is equivalent [15,23] to the assumption that the creep compliance $J(t)$ is a Bernstein function [27. This assumption is crucial for the existence of the function $g$. The assumption that the wavefront speed $c_{0}$ is finite is equivalent to the inequality $J_{0}:=J(0)>0$. If $\lim _{t \rightarrow 0} g(t)<\infty$, then Green's function has a jump discontinuity at $|x|=c_{0} t$.

It will also be shown that under some additional assumptions valid for many explicit models, viscoelastic Green's function is bounded by $C \exp \left(-g\left(t-|x| / c_{0}\right)|x|\right)$ for $t>$ $|x| / c_{0}$, where $C$ is some constant.

These results provide an estimate of Green's function near the wavefront and answer the question whether the medium supports propagation of discontinuities at the wavefront. The methods developed in this paper and in [10] are applied in [12] to two examples of viscoelastic media which support discontinuity waves.

2. Mathematical preliminaries. We shall consider the Initial-Value Problem (IVP)

$$
\begin{gathered}
\rho u_{, t t}=\nabla \cdot\left[G(t) * \nabla u_{, t}\right]+\delta(x) \delta(t), \quad t \geq 0, \quad x \in \mathbb{R} \\
u(0, x)=0 ; \quad u_{, t}(0, x)=0
\end{gathered}
$$

for the particle velocity $u$ in a hereditary viscoelastic medium. Duhamel's principle holds for equation (1); hence the above IVP is equivalent to the IVP

$$
\begin{gathered}
\rho u_{, t t}=\nabla \cdot\left[G(t) * \nabla u_{, t}\right], \quad t \geq 0, \quad x \in \mathbb{R} \\
u(0, x)=0 ; \quad u_{, t}(0, x)=\delta(x) / \rho .
\end{gathered}
$$

Since we are going to consider Green's functions with jump discontinuities, the field $u(t, x)$ will be considered as the velocity field.

It is assumed that the relaxation modulus $G(t)$ (defined for $t>0$ ) is completely monotonic; i.e. it has derivatives $\mathrm{D}^{n} G$ of arbitrary order, and these derivatives satisfy the inequalities

$$
(-1)^{n} \mathrm{D}^{n} G(t) \geq 0 \quad \text { on } \mathbb{R} \text { for } n=0,1,2 \ldots
$$

It is also assumed that $G$ is locally integrable or, equivalently,

$$
\int_{0}^{1} G(s) \mathrm{d} s<\infty
$$

In short, $G$ is LICM. It follows [15] that the creep compliance $J(t)(t \geq 0)$, related to the relaxation modulus $G(t)$ by the equation

$$
\int_{0}^{t} G(s) J(t-s) \mathrm{d} s=t \quad \text { for } t \geq 0,
$$


is a Bernstein function (BF); i.e. it is non-negative, differentiable and its derivative $J^{\prime}$ is LICM. Conversely, for a given BF $J$ equation (5) has a unique solution $G$ and $G$ is an LICM function [15. We also recall that $J(t)$ tends to a finite limit $J_{0}$ as $t \rightarrow 0$, $0 \leq J_{0}<\infty$ and $J_{0}=0$ if and only if $\lim _{t \rightarrow 0+} G(t)=\infty$.

The solution of the IVP (1) 2) is given by the formula

$$
u(t, x)=\frac{1}{4 \pi \mathrm{i}} \int_{-\mathrm{i} \infty+\varepsilon}^{\mathrm{i} \infty+\varepsilon} \frac{\kappa(p)}{2 \rho p^{2}} \mathrm{e}^{p t-\kappa(p)|x|} \mathrm{d} p
$$

where

and $\varepsilon>0$.

$$
\kappa(p):=\rho^{1 / 2} p[p \tilde{J}(p)]^{1 / 2}
$$

In [10,16] it was shown that $\kappa(p)$ is a complete Bernstein function [19, 27, i.e.

$$
\kappa(p)=p^{2} \tilde{F}(p),
$$

where $F$ is a Bernstein function. Consequently $\kappa$ has an integral representation of the form 10

$$
\kappa(p)=\frac{p}{c_{0}}+p \int_{] 0, \infty[} \frac{\nu(\mathrm{d} r)}{p+r}
$$

where $\nu$ is a positive Radon measure satisfying the inequality

$$
\int_{] 0, \infty[} \frac{\nu(\mathrm{d} r)}{1+r}<\infty
$$

and $c_{0}$ is a constant satisfying the inequalities $0<c_{0} \leq \infty$, defined by the formula

$$
1 / c_{0}:=\rho^{1 / 2} \lim _{p \rightarrow \infty}[p \tilde{J}(p)]^{1 / 2}=\left[\rho J_{0}\right]^{1 / 2} .
$$

The physical dimension of $\kappa(p)$ and $\nu(\mathrm{d} r)$ is $1 / \mathrm{L}$.

If $J_{0}>0$, then the constant $c_{0}$ is finite and it defines the wavefronts $|x|=c_{0} t$ such that $u(t, x)=0$ for $t>|x| / c_{0}$; otherwise $c_{0}=\infty$ and the solution $u(t, x)$ does not vanish anywhere in the space-time. The wavefront speed $c_{0}$ has the dimension $\mathrm{L} / \mathrm{T}$ [10]. In this paper we shall be interested in the case of $c_{0}<\infty$.

Recall that every LICM function $\varphi$ has the integral representation

$$
\varphi(t)=a+\int_{] 0, \infty[} \mathrm{e}^{-r t} \nu(\mathrm{d} r)
$$

where $\nu$ is a positive Radon measure satisfying the inequality (10) [9]. Define the function $g$ by the formula

$$
g(t)=\int_{] 0, \infty[} \mathrm{e}^{-r t} \nu(\mathrm{d} r)
$$

where the Radon measure $\nu$ is defined by equation (91). We then have an important formula

$$
\kappa(p)=p / c_{0}+p \tilde{g}(p) .
$$

By Bernstein's Theorem and (10) the function $g$ is LICM and $\lim _{t \rightarrow \infty} g(t)=0$. The dimension of $g(t)$ is $1 / \mathrm{L}$. The function $g(t)$ assumes a finite value at 0 if $\nu$ has a finite mass. Note that any function $\kappa$ given by equation (14), where $g$ is an LICM function, 
is a complete Bernstein function. Indeed, equation (8) holds for the Bernstein function $F(t)=1 / c_{0}+\int_{0}^{t} g(s) \mathrm{d} s$.

The Radon measure $\nu$ can be calculated using equation (9). If $\nu(\mathrm{d} r)=h(r) \mathrm{d} r$, then

$$
h(r)=\frac{1}{\pi} \mathfrak{I}[\kappa(p) / p]_{p=r \exp (-\mathrm{i} \pi)}
$$

[10, 16] or, using equation (7),

$$
h(r)=\frac{\rho^{1 / 2}}{\pi} \mathfrak{I}\left\{[p \tilde{J}(p)]^{1 / 2}\right\} .
$$

Note that $\kappa(p)=p / c_{0}+p^{2} \tilde{F}(p)$, where $F(t)=\int_{0}^{t} g(s) \mathrm{d} s(t \geq 0)$. Since $g \in$ $\mathcal{L}_{\text {loc }}^{1}([0, \infty[)$, the function $F(t)$ is continuous on $[0, \infty[$ and $F(0)=0$. The function $F(t)$ can therefore be extended to a continuous causal function $\Phi(t)$ on $\mathbb{R}$, and therefore it is a causal distribution. The distributional derivative $\phi$ of $\Phi$ is a causal distribution which coincides with the function $g(t)$ on $] 0, \infty[$. The analytic continuation of $\kappa$ to the imaginary axis is the Fourier transform of a causal distribution $D^{2} \Psi(t)$ where $\Psi(t):=\theta(t) / c_{0}+\Phi(t)$; hence the real part $\mathcal{A}(\omega):=\mathfrak{R} \kappa(-\mathrm{i} \omega)$ of $\kappa(-\mathrm{i} \omega)$ (the attenuation function) and its imaginary part $\mathfrak{I} \kappa(-\mathrm{i} \omega)=:-\mathcal{D}(\omega)(\mathcal{D}$ is the dispersion function) are related by the Kramers-Kronig dispersion relations [16].

In this paper the function $g(t)$ will be used to obtain asymptotic estimates and bounds for the solution $u(t, x)$ of the IVP (1)-(2). It will also be seen that $u(t, x)$ has a discontinuity at the wavefront if and only if $g(0+):=\lim _{t \rightarrow 0+} g(t)$ is finite. The function $g$ is bounded at 0 if the attenuation-dispersion measure $\nu$ has finite mass; in this case $g(0+)$ is equal to the total mass of $\nu$. This condition will turn out to be equivalent also to the inequality $J^{\prime}(0)<\infty$. The amplitude of the discontinuity will be expressed in terms of $g(0+)$ and also by $\lim _{t \rightarrow 0+} J^{\prime}(t)$.

The function $g(t)$ will also be applied to derive an upper bound for the solution near the wavefront when $g(0+)=\infty$ and the solution $u(t, x)$ is continuous at the wavefront for $t>0$.

3. Asymptotic behavior of Green's function near the wavefront. In this section an asymptotic estimate of Green's function near the wavefront will be derived.

Theorem 3.1. The function $x f(x)$ on $\mathbb{R}_{+}$is CM if and only if there is a non-negative right-continuous non-decreasing real function $g$ vanishing on $]-\infty, 0[$ such that $f=\tilde{g}$.

Proof. If $f=\tilde{g}$, then

$$
x f(x)=-\int_{0}^{\infty} \frac{\partial \mathrm{e}^{-x y}}{\partial y} g(y) \mathrm{d} y=g(0+)+\int_{0}^{\infty} \mathrm{e}^{-x y} \mathrm{~d} g(y) .
$$

Define the Radon measure $\mu$ by the formula $\mu(] 0, y])=g(y)-g(0+)$. The measure $\mu$ is positive; hence the Bernstein Theorem implies that $x f(x)$ is CM.

If $x f(x)$ is CM, then, by the Bernstein Theorem, there is a positive Radon measure $\mu$ on $\overline{\mathbb{R}_{+}}$such that

$$
x f(x)=\mu(\{0\})+\int_{] 0, \infty[} \mathrm{e}^{-x y} \mu(\mathrm{d} y) .
$$


If $g(y)=0$ for $y<0, g(0+)=\mu(\{0\})$ and $g(y)=\mu(] 0, y])$ for $y>0$, then $g(y) \geq 0, g$ is non-decreasing and $x f(x)=g(0+)+\int_{] 0, \infty[} \mathrm{e}^{-x y} \mathrm{~d} g(y)=x \int_{0}^{\infty} \mathrm{e}^{-x y} g(y) \mathrm{d} y$.

Lemma 3.2. If $(-1)^{n} f^{(n)}(x) \geq 0$ for $n=1,2, \ldots$, then

$$
(-1)^{n} \frac{\mathrm{d}^{n}}{\mathrm{~d} x^{n}} \mathrm{e}^{f(x)} \geq 0 \quad \text { for } n=0,1,2, \ldots
$$
0 .

Proof. The thesis is obviously true for $n=0$ and for $n=1$ : $\mathrm{d}^{f(x)} / \mathrm{d} x=f^{\prime}(x) \mathrm{e}^{f(x)} \leq$

We now assume that for a fixed $n \geq 1$ the identity $\mathrm{d}^{n} \mathrm{e}^{f(x)} / \mathrm{d} x^{n}=P_{n}(x) \mathrm{e}^{f(x)}$ holds, where $P_{n}(x)$ is a polynomial in the derivatives of $f$ with positive coefficients such that the sum of the orders of all the derivatives in a monomial of $P_{n}$ is $n$. The assumption is certainly true for $n=1$. From this assumption it follows that $\mathrm{d}^{n+1} \mathrm{e}^{f(x)} / \mathrm{d} x^{n+1}=$ $P_{n+1}(x) \mathrm{e}^{f(x)}$, where $P_{n+1}(x):=P_{n}^{\prime}(x)+f^{\prime}(x) P_{n}(x)$. The function $P_{n+1}$ is a sum of products of derivatives of $f$ whose orders sum up to $n+1$ with positive coefficients. We have thus proved that our assumption is true for all integers $n \geq 1$. But the hypothesis of the lemma implies that $(-1)^{n} P_{n}(x) \geq 0$, which proves the lemma.

Lemma 3.3. If $\varphi: \mathbb{R}_{+} \rightarrow \mathbb{R}$ is differentiable, non-negative and non-increasing, $\lim _{t \rightarrow 0+}[t \varphi(t)] \rightarrow 0$ and the Laplace transform $\tilde{\varphi}(p)$ of $\varphi$ exists for $p>0$, then $\exp (-p \tilde{\varphi}(p)) / p$ is the Laplace function of a non-negative non-decreasing function $H$ : $\mathbb{R}_{+} \rightarrow \mathbb{R}$.

Proof. We shall first prove that $(-1)^{n} \mathrm{~d}^{n}[p \tilde{\varphi}(p)] / \mathrm{d} p^{n} \leq 0$ for $n=1,2, \ldots$ We begin with $n=1$. Let $\varepsilon>0$. Then

$$
\begin{aligned}
\frac{\mathrm{d}}{\mathrm{d} p}\left[p \int_{\varepsilon}^{\infty} \varphi(t) \mathrm{e}^{-p t} \mathrm{~d} t\right]=-\frac{\mathrm{d}}{\mathrm{d} p}\left[\int_{\varepsilon}^{\infty} \varphi(t) \mathrm{de}^{-p t}\right] \\
\quad=\frac{\mathrm{d}}{\mathrm{d} p} \int_{\varepsilon}^{\infty} \varphi^{\prime}(t) \mathrm{e}^{-p t} \mathrm{~d} t-\frac{\mathrm{d}}{\mathrm{d} p}\left[\varphi(t) \mathrm{e}^{-p t}\right]_{t=\varepsilon}^{\infty}=-\int_{\varepsilon} t \varphi^{\prime}(t) \mathrm{e}^{-p t} \mathrm{~d} t+\varepsilon \varphi(\varepsilon) .
\end{aligned}
$$

Hence

$$
\frac{\mathrm{d}}{\mathrm{d} p}[p \tilde{\varphi}(p)]=-\int_{0}^{\infty} t \varphi^{\prime}(t) \mathrm{e}^{-p t} \mathrm{~d} t .
$$

In particular we have also proved that $t \varphi^{\prime}(t)$ is locally integrable on $[0, \infty[$.

For arbitrary $n \geq 1$ we note that

$$
\frac{\mathrm{d}^{n}}{\mathrm{~d} p^{n}}[p \tilde{\varphi}(p)]=-\frac{\mathrm{d}^{n-1}}{\mathrm{~d} p^{n-1}} \int_{0}^{\infty} t \varphi^{\prime}(t) \mathrm{e}^{-p t} \mathrm{~d} t=(-1)^{n} \int_{0}^{\infty} t^{n} \varphi^{\prime}(t) \mathrm{e}^{-p t} \mathrm{~d} t .
$$

But $\varphi^{\prime}(t) \leq 0$ almost everywhere, hence

$$
(-1)^{n} \frac{\mathrm{d}^{n}}{\mathrm{~d} p^{n}}[p \tilde{\varphi}(p)] \leq 0, \quad n=1,2, \ldots
$$

Lemma 3.2 implies that

$$
(-1)^{n} \frac{\mathrm{d}^{n}}{\mathrm{~d} p^{n}} \mathrm{e}^{-p \tilde{\varphi}(p)} \geq 0 \quad \text { for } n=0,1,2, \ldots
$$


Theorem 3.1 now implies that

$$
\mathrm{e}^{-p \tilde{\varphi}(p)} / p=\int_{0}^{\infty} \mathrm{e}^{-p t} H(t) \mathrm{d} t
$$

where the function $H$ is non-negative and non-decreasing.

Corollary 3.4. If $\varphi(0+)=\lim _{t \rightarrow 0+} \varphi(t)$ exists, then

$$
\lim _{p \rightarrow \infty} \mathrm{e}^{-p \tilde{\varphi}(p)}=\exp \left(-\lim _{p \rightarrow \infty}[p \tilde{\varphi}(p)]\right)=\mathrm{e}^{-\varphi(0+)},
$$

and the limit $H(0+)$ exists and equals $\mathrm{e}^{-\varphi(0+)}$.

Similarly, if the limit $\varphi_{\infty}=\lim _{t \rightarrow \infty} \varphi(t)$ exists, then $\lim _{t \rightarrow \infty} H(t)=\mathrm{e}^{-\varphi_{\infty}}$.

Lemma 3.5. If the function $\varphi \in \mathcal{L}_{\text {loc }}^{1}\left(\mathbb{R}_{+}\right)$is non-negative, non-increasing and convex, and $\lim _{t \rightarrow 0+}\left[t \varphi^{\prime}(t)\right]=0$, then $\lim _{p \rightarrow \infty}[p \tilde{\varphi}(p)-\varphi(1 / p)]=0$.

Proof. The Laplace transform $\tilde{\varphi}$ of $\varphi$ is defined and

$$
\begin{aligned}
p \tilde{\varphi}(p)-\varphi(1 / p)=p \int_{0}^{\infty} \varphi(t) \mathrm{e}^{-p t} \mathrm{~d} t-\varphi(1 / p) & \\
& =\int_{0}^{\infty}[\varphi(t / p)-\varphi(1 / p)] \mathrm{e}^{-t} \mathrm{~d} t=I_{1}+I_{2} .
\end{aligned}
$$

We begin with an estimate of $I_{1}=\int_{0}^{1}[\varphi(t / p)-\varphi(1 / p)] \mathrm{e}^{-t} \mathrm{~d} t$. The function $\varphi$ is non-decreasing and $t \leq 1$, the integrand is non-negative, hence $I_{1} \geq 0$. On the other hand,

$$
\begin{aligned}
I_{1} \leq \int_{0}^{1}[\varphi(t / p) & -\varphi(1 / p)] \mathrm{d} t=p \int_{0}^{1 / p} \varphi(q) \mathrm{d} q-\varphi(1 / p)=-p \int_{0}^{1 / p} q \varphi^{\prime}(q) \mathrm{d} q \\
= & -p \int_{0}^{1 / p} q \varphi^{\prime}(q) \mathrm{d} q+[p q \varphi(q)]_{q=0}^{q=1 / p}-\varphi(1 / p)=-p \int_{0}^{1 / p} q \varphi^{\prime}(q) \mathrm{d} q .
\end{aligned}
$$

By Rolle's Theorem there is a $q_{1} \in[0,1 / p]$ such that

$$
I_{1} \leq-q_{1} \varphi^{\prime}\left(q_{1}\right) .
$$

If $p \rightarrow \infty$, then $q_{1} \rightarrow 0$ and, by assumption, $q_{1} \varphi^{\prime}\left(q_{1}\right) \rightarrow 0$. Consequently $I_{1} \rightarrow 0$ as $p \rightarrow \infty$.

We now turn to $I_{2}:=\int_{1}^{\infty}[\varphi(t / p)-\varphi(1 / p)] \mathrm{d} t$. For $t>1$ we have $0 \leq \varphi(1 / p)-$ $\varphi(t / p)=\int_{1 / p}^{t / p}\left|\varphi^{\prime}(q)\right| \mathrm{d} q$. Convexity of $\varphi$ implies that $\left|\varphi^{\prime}(q)\right|$ is non-increasing, hence $0 \leq \varphi(1 / p)-\varphi(t / p) \leq(t / p)\left|\varphi^{\prime}(1 / p)\right|$. Hence

$$
0 \leq-I_{2} \leq \frac{1}{p}\left|\varphi^{\prime}(1 / p)\right| \int_{1}^{\infty} t \mathrm{e}^{-t} \mathrm{~d} t .
$$

On account of our assumptions the first factor on the right-hand side tends to 0 as $p \rightarrow \infty$. Hence $I_{2} \rightarrow 0$ as $p \rightarrow \infty$, which proves the thesis.

Lemma 3.6. Let $0<\lambda \leq 1$. If $\varphi$ is non-negative, differentiable, non-increasing and convex and $t \varphi^{\prime}(t) \rightarrow 0$ for $t \rightarrow 0$, then $0 \leq \varphi(\lambda / p)-\varphi(1 / p) \rightarrow 0$ as $p \rightarrow \infty$. 
Proof.

$$
\begin{aligned}
0 \leq \varphi(\lambda / p)-\varphi(1 / p)=\int_{\lambda / p}^{1 / p}\left|\varphi^{\prime}(q)\right| \mathrm{d} q \leq \frac{1-\lambda}{p} \mid \varphi^{\prime} & (\lambda / p) \mid \\
& \leq \frac{1}{p}\left|\varphi^{\prime}(\lambda / p)\right|=\frac{1}{\lambda} \frac{\lambda}{p}\left|\varphi^{\prime}(\lambda / p)\right| \rightarrow 0
\end{aligned}
$$

in view of the last hypothesis.

We are now ready to investigate the asymptotic behavior of the function $H(t, r)$ at $t=0$.

THEOREM 3.7.

$$
H(t, r) \sim_{t \rightarrow 0} \mathrm{e}^{-g(t) r}, \quad t>0 .
$$

Proof. The Laplace transform of $H(t, r)$ is $\exp (-p \tilde{\varphi}(p)) / p$, where $\varphi(t)=g(t) r$. We now consider the limit at $t \rightarrow 0$ of the ratio

$$
R:=\exp (-(p / \lambda) \tilde{\varphi}(p / \lambda)) / \exp (-p \tilde{\varphi}(p)),
$$

where $\lambda>0$ is arbitrary. Lemma 3.6 implies that the limit of $\mathrm{R}$ at $p=\infty$ will not change if we divide it by $\mathrm{e}^{-\varphi(\lambda / p)+\varphi(1 / p)}$. Hence

$$
\lim _{p \rightarrow \infty} R=\lim _{p \rightarrow \infty} \exp (-p / \lambda \tilde{\varphi}(p / \lambda)+\varphi(\lambda / p)) \times \lim _{p \rightarrow \infty} \exp (p \tilde{\varphi}(p)-\varphi(1 / p)) .
$$

Lemma 3.5 implies that both limits on the right-hand side are equal to 1 , hence $\lim _{p \rightarrow \infty} R=1$ for all $\lambda>0$. Consequently the function $l(p):=\exp (-p \tilde{\varphi}(p))$ is slowly varying at infinity.

But $\tilde{H}(p, r)=l(p) / p$ and by the Karamata Tauberian theorem (Appendix) $H(t, r) \sim_{0}$ $l(1 / t) \equiv \mathrm{e}^{-\tilde{\varphi}(1 / t) / t}$. By Lemma 3.5 $H(t, r) \sim_{0} \mathrm{e}^{-\varphi(t)} \equiv \mathrm{e}^{-g(t) r}$.

It remains to link the estimates of $H(t, r)$ to Green's function.

THEOREM 3.8. Let

$$
f(t)=\int_{0}^{t} g(s) \mathrm{d} s .
$$

Then

$$
u(t, x)=\frac{1}{2 \rho}\left[H\left(t-|x| / c_{0}\right)+f(t) * H\left(t-|x| / c_{0}\right)\right]
$$

and

$$
u(t, x)=\frac{1}{2 \rho} H\left(t-|x| / c_{0}\right)\left[1+\mathrm{O}\left[t-|x| / c_{0}\right] .\right.
$$

Proof. The function $f$ is well-defined because $g \in \mathcal{L}_{\text {loc }}^{1}([0, \infty[)$ and $f(t) \geq 0, f(0)=0$. It follows from equations (6) and (14) that

$$
\tilde{u}(p, x)=\frac{\kappa(p)}{2 \rho p^{2}} \mathrm{e}^{-p|x| / c_{0}} \mathrm{e}^{-p \tilde{g}(p)|x|} .
$$

But $\kappa(p) \sim_{\infty} p / c_{0}$ and

$$
\frac{\kappa(p)}{p^{2}}-\frac{1}{p c_{0}}=\frac{\tilde{g}(p)}{p} .
$$

This proves equation (21). 
The function $H(\cdot, r)$ is non-decreasing, hence

$f(t) * H\left(t-r / c_{0}\right)=\int_{0}^{t-r / c_{0}} f(s) H\left(t-s-r / c_{0}\right) \mathrm{d} s \leq H\left(t-r / c_{0}\right) \int_{0}^{t-r / c_{0}} f(s) \mathrm{d} s$.

The integral on the right-hand side is $\mathrm{O}\left[\left(t-r / c_{0}\right)\right]$. This ends the proof.

For $\left|t-r / c_{0}\right| \leq C_{1}$ the second term in the brackets is bounded from above by a number $C_{2}$; hence $|u(t, x)| \leq C H\left(t-|x| / c_{0}\right)$ for some constant $C>0$. The last equation can be used to determine upper bounds on Green's function near the wavefront.

The function $g\left(t-r / c_{0}\right) r$ is a local phase of Green's function near its wavefront.

4. Upper bound for Green's function near a wavefront in media with unbounded $g(t)$. We recall that Green's functions of media with unbounded $g(t)$ do not have discontinuities.

Lemma 4.1. If the function $\varphi(t)$ is non-increasing and differentiable, $\lim _{t \rightarrow 0}[t \varphi(t)]=0$, $\varphi(t) \geq A$ for some real constant $A$, and the function $-t \varphi^{\prime}(t)$ is non-increasing, then the inverse Laplace transform of $\exp (-p \tilde{\varphi}(p)) / p$ is bounded from above by $\exp (-\varphi(t))$.

Proof. Let $f(t)$ denote the inverse Laplace transform of $\exp (-p \tilde{\varphi}(p))$. Then $\exp (-p \tilde{\varphi}(p)) / p$ is the Laplace transform of $F(t):=\int_{0}^{t} f(s) \mathrm{d} s$. The limit $\lim _{t \rightarrow \infty} F(t)$ $=\int_{0}^{\infty} f(s) \mathrm{d} s$ is finite:

$$
\begin{aligned}
& \lim _{t \rightarrow \infty} F(t)=\lim _{p \rightarrow 0} \mathrm{e}^{-p \tilde{\varphi}(p)}=\exp \left(-\lim _{p \rightarrow 0}[p \tilde{\varphi}(p)]\right)=\exp \left(-\lim _{t \rightarrow \infty} \varphi(t)\right) \\
& \leq \mathrm{e}^{-A}<\infty
\end{aligned}
$$

We now note the identity

$$
t f(t)=-\int_{0}^{t} s \varphi(s) f(t-s) \mathrm{d} s .
$$

Indeed, the Laplace transform of the left-hand side is

$$
\int_{0}^{\infty} t f(t) \mathrm{e}^{-p t} \mathrm{~d} t=-\frac{\mathrm{d} \tilde{f}(p)}{\mathrm{d} p}=\left[p \frac{\mathrm{d} \tilde{\varphi}(p)}{\mathrm{d} p}+\tilde{\varphi}(p)\right] \tilde{f}(p),
$$

while the Laplace transform of the right-hand side is

$$
-\left(t \varphi^{\prime}(t)\right)^{\sim} \tilde{f}(p)=-\left\{\left[(t \varphi(t))^{\prime}\right]^{\sim}-\tilde{\varphi}(p)\right\} \tilde{f}(p)=\tilde{f}(p)[p \mathrm{~d} \tilde{\varphi}(p) / \mathrm{d} p+\varphi(p)] ;
$$

hence the two are equal. Equation (24) follows from the uniqueness theorems for the Laplace transform.

The assumption that $-t \varphi^{\prime}(t)$ is non-increasing implies that $t f(t) \geq-t \varphi(t) \int_{0}^{t} f(s) \mathrm{d} s$, hence

$$
-\varphi^{\prime}(t) \leq \frac{\mathrm{d}}{\mathrm{d} t} \ln (F(t))
$$

Integration of this inequality over $[t, \infty[$ yields the inequality $\varphi(t) \leq-\ln (F(t))$ on account of equation (23), hence $F(t) \leq \mathrm{e}^{-\varphi(t)}$. 
Theorem 4.2. Let the function $g$ defined by equation (13) be such that $-t g^{\prime}(t)$ is nonincreasing. For $t-|x| / c_{0}<\varepsilon$ there is a constant $C$ such that

$$
u(t, x) \leq C \exp \left(-g\left(t-|x| / c_{0}\right)|x|\right) .
$$

Proof. The function $g$ defined by equation (13) is LICM; hence it is non-increasing and $-t g^{\prime}(t) \geq 0$. It therefore has a limit at 0 which is either finite or $\infty$.

By Theorem 3.8 for $0 \leq t-|x| / c_{0} \leq C_{1}$, where $C_{1}$ is some constant,

$$
u(t, x) \leq C H\left(t-|x| / c_{0},|x|\right)
$$

where $H(t, r)$ is the inverse Laplace transform of $\exp (-p \tilde{g}(p) r) / p$. By Lemma 4.1 $H\left(t-r / c_{0}, r\right) \leq \exp \left(-r g\left(t-r / c_{0}\right)\right)$.

EXAMPLES.

(1) If $\kappa(p)-p / c_{0} \equiv p \tilde{g}(p)=a p^{\alpha}$, where $0<\alpha<1$ and $a>0$, then $g(t)=a t^{-\alpha} /$ $\Gamma(1-\alpha)$ is LICM and the function $-t g^{\prime}(t)$ is non-increasing. Hence equation (26) holds. Consequently $u(p, x) \leq C \exp \left(-a\left(t-|x| / c_{0}\right)^{-\alpha}\right)$.

(2) $g(t):=b \ln (1 /(a t)+A)$, where $a, b>0$ have the dimensions $1 / \mathrm{T}$ and $1 / \mathrm{L}, A \geq 1$, is LICM because

$$
\ln (1 /(a t)+A)=\ln (A)+\int_{0}^{\infty} \frac{1-\mathrm{e}^{-r}}{r} \mathrm{e}^{-r t /(a A)} \mathrm{d} r
$$

and the second term is the Laplace transform of a positive function satisfying equation (10). The identity (27) can be checked by differentiating both sides with respect to $t$ and working out the resulting integral on the right-hand side. Consequently $\kappa(p)=p / c_{0}+p \tilde{g}(p)$, where $c_{0}>0$, is a complete Bernstein function. The function $-t g^{\prime}(t)$ is non-increasing. Hence equation (26) holds and

$$
H(t, r) \leq(1 /(a t)+A)^{-b r} .
$$

Note that the wavefront singularity decreases stepwise by the formula $H\left(t-r / c_{0}\right)$ $\sim_{t \rightarrow\left(r / c_{0}\right)+} C_{1}\left(t-r / c_{0}\right)^{r b}$

(3) The LICM functions $(t+1)^{-1}$ and $\mathrm{e}^{-t}$ do not satisfy the hypotheses of Theorem 4.2 .

REMARK 4.3. If $\kappa(p)-p / c_{0}$ is regularly varying with index $\left.\alpha \in\right] 0,1[$ at infinity, i.e. $\kappa(p)-p / c_{0} \equiv p \tilde{g}(p) \sim_{\infty} p^{\alpha} l(p)$, where the function $l$ is slowly varying at infinity, then by the Karamata Tauberian theorem (Appendix) $g(t) \sim_{0} l(1 / t) t^{-\alpha} / \Gamma(1-\alpha) . g$ is nonincreasing, but the hypothesis that $-t g^{\prime}(t)$ is non-increasing need not be satisfied. The function $g(t)=B \ln (1 /(a t)+A) t^{-\alpha} / \Gamma(1-\alpha)$ is however LICM (because it is a product of two CM functions) and satisfies the conditions of Theorem 4.2 Consequently

$$
|u(t, x)| \leq\left[1 /\left[a\left(t-|x| / c_{0}\right)\right]+A\right]^{-B|x|\left(t-|x| / c_{0}\right)^{-\alpha}} .
$$

5. Relations between the function $g$ and the creep rate function. We shall show that the value of $g(0+)$ or the singularity of the function $g$ at 0 are to some extent determined by the value of the creep rate function at 0 or its singularity at 0 . We shall thus relate the wavefront jump discontinuity or regularity of Green's function at the wavefront to the asymptotics of creep rate at $t=0$.

If $g(0+)<\infty$, then Corollary 3.4 implies that $\lim _{t \rightarrow 0+} H(t, r)=\lim _{p \rightarrow \infty} \mathrm{e}^{-p \tilde{g}(p) r}=$

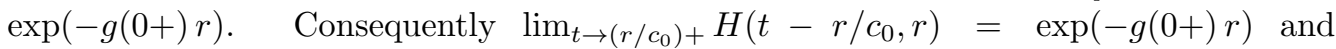


$\lim _{t \rightarrow\left(r / c_{0}\right)-} H\left(t-r / c_{0}, r\right)=0$. Thus the wavefront carries a discontinuity of Green's function. The wavefront discontinuity can be expressed in terms of $J^{\prime}(0+)$.

Theorem 5.1. If $J^{\prime}$ has a finite limit at 0 , then the function $g$ also has a finite limit at 0 and

$$
g(0+)=\rho c_{0} J^{\prime}(0+) / 2 .
$$

Furthermore

$$
g(t) \leq \rho c_{0} J^{\prime}(t) / 2
$$

Proof. Since $\kappa(p)=p / c_{0}+p \tilde{g}(p)$ and $\kappa(p)=\rho^{1 / 2} p[p \tilde{J}(p)]^{1 / 2}=\rho^{1 / 2} p\left[J_{0}+\widetilde{J}^{\prime}(p)\right]^{1 / 2}$, using the formula $c_{0}=1 /\left(\rho J_{0}\right)^{1 / 2}$,

$$
\frac{2}{c_{0}} p \tilde{g}(p)+p[\tilde{g}(p)]^{2}=\rho p \widetilde{J}^{\prime}(p) .
$$

Since $J^{\prime}(0+)=\lim _{p \rightarrow \infty}\left[p \widetilde{J}^{\prime}(p)\right]$ is finite and $p[\tilde{g}(p)]^{2} \geq 0$, the function $p \tilde{g}(p)$ is bounded from above. It follows from the proof of Lemma 3.3 that this function is non-decreasing; hence it tends to a limit $A$ as $p \rightarrow \infty$. Hence the second term on the left-hand side of equation (30) tends asymptotically to $A^{2} / p$ for large $p$, and therefore it tends to zero as $p$ tends to infinity. Consequently $A=\rho c_{0} J^{\prime}(0) / 2$, which proves the first part of the thesis.

For the second part, we note that $\tilde{g}(p)$ is the Laplace transform of a non-negative function; hence Theorem 3.1 implies that $p \tilde{g}(p)$ is a CM function. The second term on the left-hand side of equation (30) is the product of three CM functions $\left(p^{-1}\right.$ and twice $p \tilde{G}(p))$; hence it is CM. Equation (28) implies that

$$
\frac{\rho c_{0}}{2} p \widetilde{J}^{\prime}(p)-p \tilde{g}(p) \equiv \int_{0}^{\infty} \frac{\mathrm{d}}{\mathrm{d} t}\left[\frac{\rho c_{0}}{2} J^{\prime}(t)-g(t)\right] \mathrm{e}^{-p t} \mathrm{~d} t
$$

By Bernstein's theorem (Appendix) the above expression is the Laplace transform of a positive Radon measure $\mu$. By the uniqueness of the Laplace transform,

and therefore

$$
\mu(\mathrm{d} t)=\frac{\mathrm{d}}{\mathrm{d} t}\left[\frac{\rho c_{0}}{2} J^{\prime}(t)-g(t)\right] \mathrm{d} t
$$

$$
\frac{\mathrm{d}}{\mathrm{d} t}\left[\frac{\rho c_{0}}{2} J^{\prime}(t)-g(t)\right] \mathrm{d} t \geq 0 .
$$

The last inequality and equation (28) imply inequality (29).

Equation (28) can also be expressed in the form

$$
g(0+)=J^{\prime}(0+) /\left(2 J_{0} c_{0}\right) .
$$

It follows from equation (5) that $J_{0} G_{0}=1$ and $J^{\prime}(0+) G_{0}+G^{\prime}(0+) J_{0}=0$, where $G_{0}=\lim _{t \rightarrow 0} G(t)<\infty$ in view of our assumption that $J_{0}>0$. The above identities imply a third expression for $g(0+)$ :

$$
g(0+)=-G^{\prime}(0+) /\left(2 \rho c_{0}^{3}\right),
$$

which is consistent with Chu's amplitude equation for a shock wave [5]. Under our assumptions $g(0+)=J^{\prime}(0+)=\infty$ if and only if $G^{\prime}(0+)=-\infty$. The latter inequality was given as a criterion for non-existence of shocks by Prüss in [25]. 
Equation (29) implies that the singularity of $g$ at 0 is not stronger than the singularity of the creep rate $J^{\prime}$.

Equations (21), (20) and (31) imply that the jump discontinuity at the wavefront is given by the expression $(2 \rho)^{-1} \exp \left(-J^{\prime}(0+) r /\left(2 J_{0} c_{0}\right)\right)$, which is a well-known result [4].

6. Conclusions. The causal function $g(t)$ introduced in [10,16] in the context of Kramers-Kronig relations has now been used to study the wavefront asymptotics of Green's functions. The function $g$ is majorized by the creep rate function $J^{\prime}$ multiplied by the factor $\rho c_{0} / 2$, and its value at 0 can be expressed in terms of $J^{\prime}(0)$. Consequently discontinuities are expected at the wavefront if and only if $J^{\prime}(0)<\infty$. Use of $g$ allows for more detailed estimates than would be possible with constitutive parameters only.

Non-linearity can generate shock waves in elastic media through gradient catastrophe. Our analysis of wavefront singularities suggests that this process might be impeded by an unbounded viscoelastic attenuation. So far little has been done to clarify the competition between non-linearity and various kinds of viscoelastic dissipation.

Appendix A. Recapitulation of necessary mathematical concepts. An infinitely differentiable function $g:] 0, \infty\left[\rightarrow \mathbb{R}\right.$ is completely monotonic $(\mathrm{CM})$ if $(-1)^{n} \mathrm{D}^{n} g(t) \geq$ 0 for $t>0$ and $n \in \mathbb{Z}_{+} \cup\{0\}$. By the Leibniz formula the product $f(t) g(t)$ of two CM functions $f$ and $g$ is CM.

The CM function $g$ can have a singularity at 0 , but it is integrable on any finite interval not including 0 . The function $g$ is therefore locally integrable if and only if $\int_{0}^{1} g(t) \mathrm{d} t<\infty$. In view of non-negativity, monotonicity and continuity $g$ always has a limit $g_{\infty} \geq 0$ at $\infty$.

Bernstein's Theorem [27] asserts that $g$ is a completely monotonic function if and only if $g$ is the Laplace transform of a positive Radon measure (essentially a locally finite measure) $\nu$ such that

$$
g(t)=\int_{[0, \infty[} \mathrm{e}^{-r t} \nu(\mathrm{d} r)
$$

$g$ has a finite limit at 0 if $\nu$ has finite mass and then $\lim _{t \rightarrow 0} g(t)=g(0+)=\nu([0, \infty[) . g$ is locally integrable if and only if $\nu$ satisfies the inequality (10) 9].

A non-negative function $f$ on $] 0, \infty$ [ is called a Bernstein function if it is differentiable and its derivative is completely monotonic. Monotonicity and continuity of $f$ imply that it has a finite non-negative limit at 0 .

A function $h$ on $] 0, \infty[$ is said to be a complete Bernstein function if it has the form $h(p)=p^{2} \tilde{F}(p)$, where $F$ is a Bernstein function. The function $h$ is a complete Bernstein function if and only if it has the integral representation

$$
h(p)=a+b p+p \int_{] 0, \infty[} \frac{\nu(\mathrm{d} r)}{p+r}
$$

where $a$ and $b$ are non-negative constants and $\nu$ is a positive Radon measure satisfying inequality (10) 27].

A measurable real function $f$ on $[0, \infty$ [ is said to be regularly varying with index $\gamma \in \mathbb{R}$ at $a=0$ or $\infty$ if $\lim _{t \rightarrow a} f(\lambda t) / f(t)=\lambda^{\gamma}$ for $\lambda>0$ [1]. The function $f$ is said to be slowly 
varying at $a$ if it is regularly varying with index 0 at $a$. A general function $f$ regularly varying with index $\gamma$ at $a$ has the form $f(t)=t^{\alpha} l(t)$, where $l(t)$ is slowly varying at $a$.

We recall the Karamata Tauberian theorem ([1], a corollary of Theorems 1.7.1 and 1.7.2):

Theorem A.1. If $f \in \mathcal{L}_{\text {loc }}^{1}([0, \infty[)$ is non-negative and monotone for $t>T$, where $T$ is a positive number, $\alpha \geq 0$ and the function $l(t)$ is slowly varying at infinity, then $f(t) \sim_{\infty} t^{\alpha-1} l(t)$ is equivalent to $\tilde{f}(p) \sim_{0} p^{-\alpha} l(1 / p)$.

\section{REFERENCES}

[1] N. H. Bingham, C. M. Goldie, and J. L. Teugels, Regular variation, Encyclopedia of Mathematics and its Applications, vol. 27, Cambridge University Press, Cambridge, 1987. MR.898871(88i:26004)

[2] M. Caputo and F. Mainardi, New dissipation model based on memory mechanism, Pure Applied Geophysics 91 (1976), 134-147. MR2382783

[3] J. M. Carcione, F. Cavallini, F. Mainardi, and A. Hanyga, Time-domain seismic modeling of constant- $Q$ wave propagation using fractional derivatives, Pure Appl. Geophys. 159 (2002), 17141736.

[4] R. M. Christensen, Theory of viscoelasticity: An introduction, Academic Press, New York, 1971.

[5] Boa-Teh Chu, Stress waves in isotropic linear viscoelastic materials. I, J. Mécanique 1 (1962), 439-462. MR0149753 (26 \#7238)

[6] W. Desch and R. C. Grimmer, Initial-boundary value problems for integro-differential equations, Integro-differential evolution equations and applications (Trento, 1984), J. Integral Equations 10 (1985), no. 1-3, suppl., 73-97. MR831236 (87f:45025)

[7] W. Desch and R. Grimmer, Smoothing properties of linear Volterra integro-differential equations, SIAM J. Math. Anal. 20 (1989), no. 1, 116-132, DOI 10.1137/0520009. MR977492 (89m:45014)

[8] J. M. Greenberg, The existence of steady shock waves for a class of nonlinear dissipative materials with memory, Quart. Appl. Math. 26 (1968), 27-34.

[9] G. Gripenberg, S.-O. Londen, and O. Staffans, Volterra integral and functional equations, Encyclopedia of Mathematics and its Applications, vol. 34, Cambridge University Press, Cambridge, 1990. MR $1050319(91 \mathrm{c}: 45003)$

[10] Andrzej Hanyga, Wave propagation in linear viscoelastic media with completely monotonic relaxation moduli, Wave Motion 50 (2013), no. 5, 909-928, DOI 10.1016/j.wavemoti.2013.03.002. MR.3070949

[11] A. Hanyga, Dispersion and attenuation for an acoustic wave equation consistent with viscoelasticity, J. Comput. Acoust. 22 (2014), no. 3, 1450006, 22 pp. MR3232047

[12] A. Hanyga, Attenuation and shock waves in linear hereditary viscoelastic media. Strick-Mainardi and Jeffreys-Lomnitz-Strick creep compliances., arXiv:1401.3094 [math-ph] (2014).

[13] A. Hanyga and M. Seredyńska, Some effects of the memory kernel singularity on wave propagation and inversion in poroelastic media, I: Forward modeling, Geophys. J. Int. 137 (1999), 319-335.

[14] A. Hanyga and M. Seredyńska, Asymptotic wavefront expansions in hereditary media with singular memory kernels, Quart. Appl. Math. LX (2002), 213-244. MR 1900491 (2004f:35177)

[15] Andrzej Hanyga and Małgorzata Seredyńska, Relations between relaxation modulus and creep compliance in anisotropic linear viscoelasticity, J. Elasticity $\mathbf{8 8}$ (2007), no. 1, 41-61, DOI 10.1007/s10659-007-9112-6. MR2337270 (2008j:74010)

[16] A. Hanyga and M. Seredyńska, Relaxation, dispersion, attenuation and finite propagation speed in viscoelastic media, J. Math. Phys. 51 (2010), no. 9, 092901-092916. MR2742817 (2011j:74067)

[17] S. Havriliak and S. Negami, A complex plane analysis of alpha-dispersions in some polymer systems, J. Polym. Sci. 14 (1966), 99-117.

[18] W. J. Hrusa and M. Renardy, On wave propagation in linear viscoelasticity, Quart. Appl. Math. 43 (1985), no. 2, 237-254. MR793532 (86j:45022)

[19] N. Jacob, Pseudo differential operators and Markov processes. Vol. I, Fourier analysis and semigroups, Imperial College Press, London, 2001. MR.1873235 (2003a:47104)

[20] E. Kjartansson, Constant Q-wave propagation and attenuation, J. Geophys. Res. 84 (1979), 47374748 . 
[21] A. A. Lokshin and Yu. V. Suvorova, Matematicheskaya teoriya rasprostraneniya voln $v$ sredakh $s$ pamyatyu (Russian), Moskov. Gos. Univ., Moscow, 1982. MR676810 (84m:73031)

[22] F. Mainardi, Fractional calculus and waves in linear viscoelasticity, World-Scientific, 2010. MR2676137(2011e:74002)

[23] A. Molinari, Viscoélasticité linéaire et functions complètement monotones, J. Mécanique 12 (1975), 541-553. MR0368558 (51 \#4799)

[24] S. P. Näsholm and S. Holm, On a fractional Zener elastic wave equation, Fract. Calc. Appl. Anal. 16 (2013), 26-50. MR3016640

[25] J. Prüss, Positivity and regularity of hyperbolic Volterra equations in Banach spaces, Math. Ann. 279 (1987), 317-344. MR0919509 (89h:45004)

[26] M. Renardy, W. J. Hrusa, and J. A. Nohel, Mathematical problems in viscoelasticity, Longman Scientific \& Technical, Essex; John Wiley, New York, 1987. MR0919738 (89b:35134)

[27] René L. Schilling, Renming Song, and Zoran Vondraček, Bernstein functions, Theory and applications, de Gruyter Studies in Mathematics, vol. 37, Walter de Gruyter \& Co., Berlin, 2010. MR.2598208(2011d:60060)

[28] Peter Straka, Mark M. Meerschaert, Robert J. McGough, and Yuzhen Zhou, Fractional wave equations with attenuation, Fract. Calc. Appl. Anal. 16 (2013), no. 1, 262-272, DOI 10.2478/s13540013-0016-9. MR.3016653

[29] E. Strick, A predicted pedestal effect for a pulse propagating in constant $Q$ solids, Geophysics 35 (1970), 387-403.

[30] E. Strick, An explanation of observed time discrepancies between continuous and conventional well velocity surveys, Geophysics 36 (1971), 285-295.

[31] P. J. Torvik and R. L. Bagley, On the appearance of the fractional derivative in the behavior of real materials, J. Appl. Mechanics 51 (1983), 294-298. 